\title{
Direct Synthesis of Lithium Intercalated Graphene for Electrochemical Energy Storage Application
}

\author{
Ashavani Kumar, ${ }^{1}$ Arava Leela Mohana Reddy, ${ }^{1,}$ Arnab Mukherjee, ${ }^{2}$ Madan Dubey, ${ }^{3}$ Xiaobo \\ Zhan, ${ }^{1}$ Neelam Singh, ${ }^{1}$ Lijie Ci, ${ }^{1}$ W. Edward Billups, ${ }^{2}$ John Nagurny, ${ }^{4}$ Gandhi Mital, ${ }^{4}$ Pulickel \\ M. Ajayan ${ }^{1,2, *}$ \\ ${ }^{1}$ Department of Mechanical Engineering and Materials Science and ${ }^{2}$ Department of Chemistry, Rice University, \\ 6100 Main St. Houston, TX 77005, USA \\ ${ }^{3}$ U.S. Army Research Laboratory, 2800 Powder Mill Rd, Adelphi, MD 20783, USA \\ ${ }^{4}$ Lockheed Martin Maritime Systems \& Sensors, 9500 Godwin Drive, Manassas, VA 20110, USA \\ "To whom correspondence should be addressed. E-mail: leela@ rice.edu, ajayan@ rice.edu
}

Section 1S: SEM and TEM microscopy were used to determine the morphology and size of the graphene domains. A representative SEM image of the dry, reduced graphite oxide is shown in Figure 1S (A and B) and reveals randomly aggregated, thin, crumpled sheets that form a disordered solid powder with a "fluffy" appearance. The fluffy structure shown in the SEM image is due to the aggregation of reduced GO that is formed after solvent evaporation and is in good agreement with previous work. ${ }^{1-3}$

The absence of charging during the SEM imaging indicates that the network of graphenebased sheets and the individual sheets are electrically conductive. The reduced GO was dispersed in hexane using ultrasonication and a drop coated film was prepared on a carbon coated grid for TEM analysis. Figures $1 \mathrm{~S}$ (B) is TEM images of reduced GO film showing a wrinkled thin paper-like structure at low magnification. 

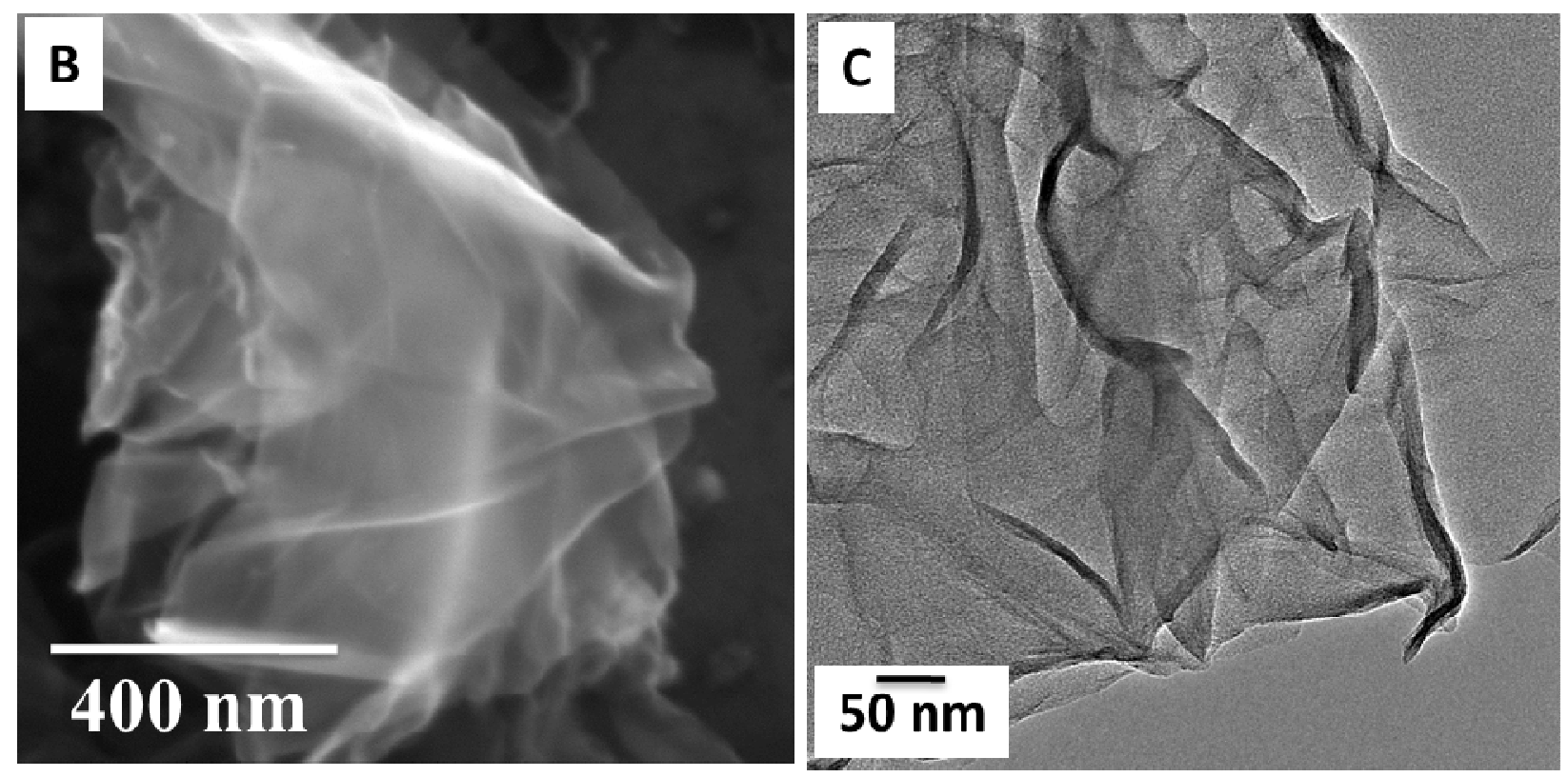

Figure 1S: (A) SEM image of drop coated film of graphene sheet on silicon (B) high magnification TEM images of a Li-RGO 


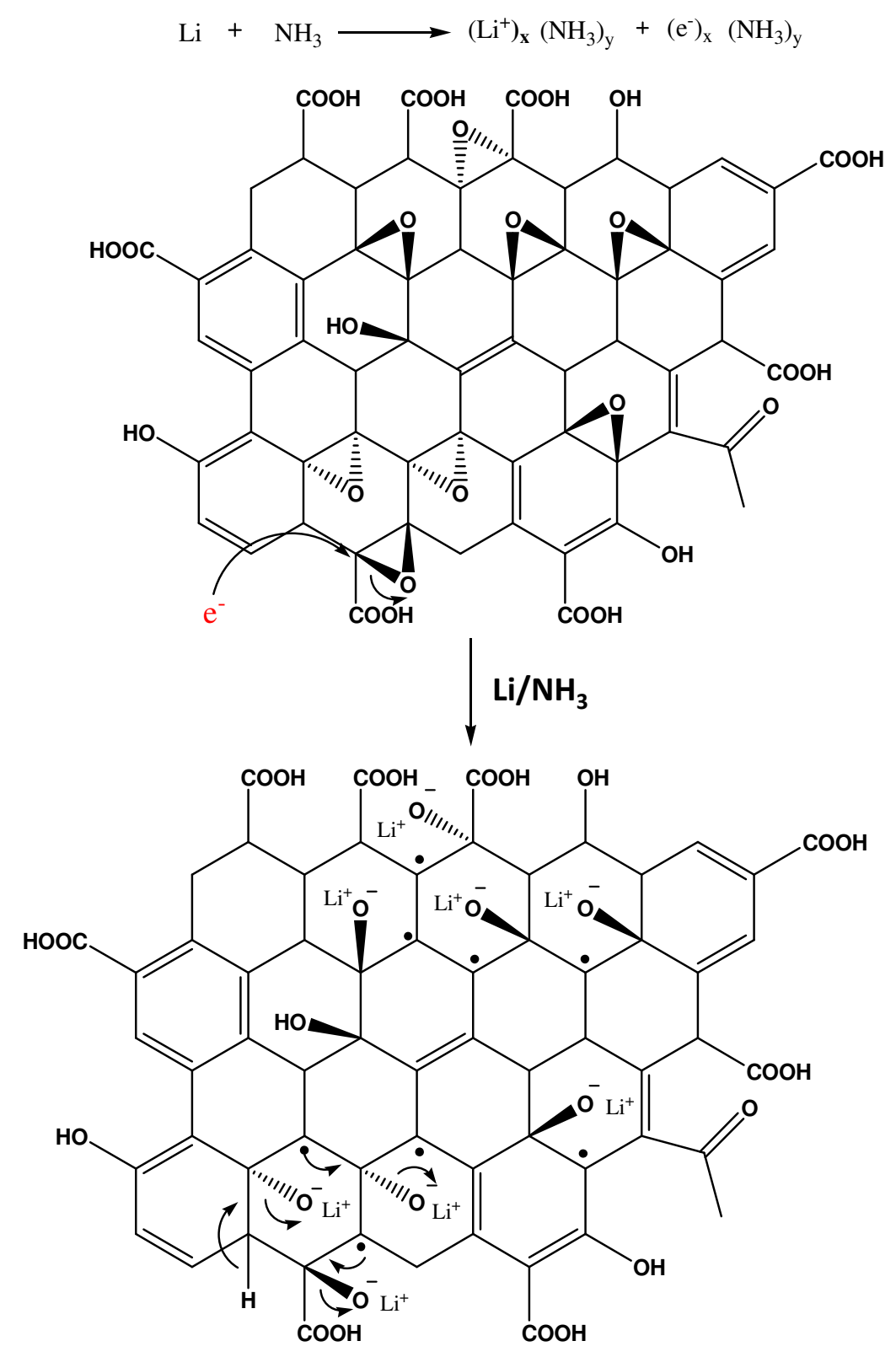

Figure 2S: Reaction mechanism of graphene oxide by lithium metal in liquid ammonia 

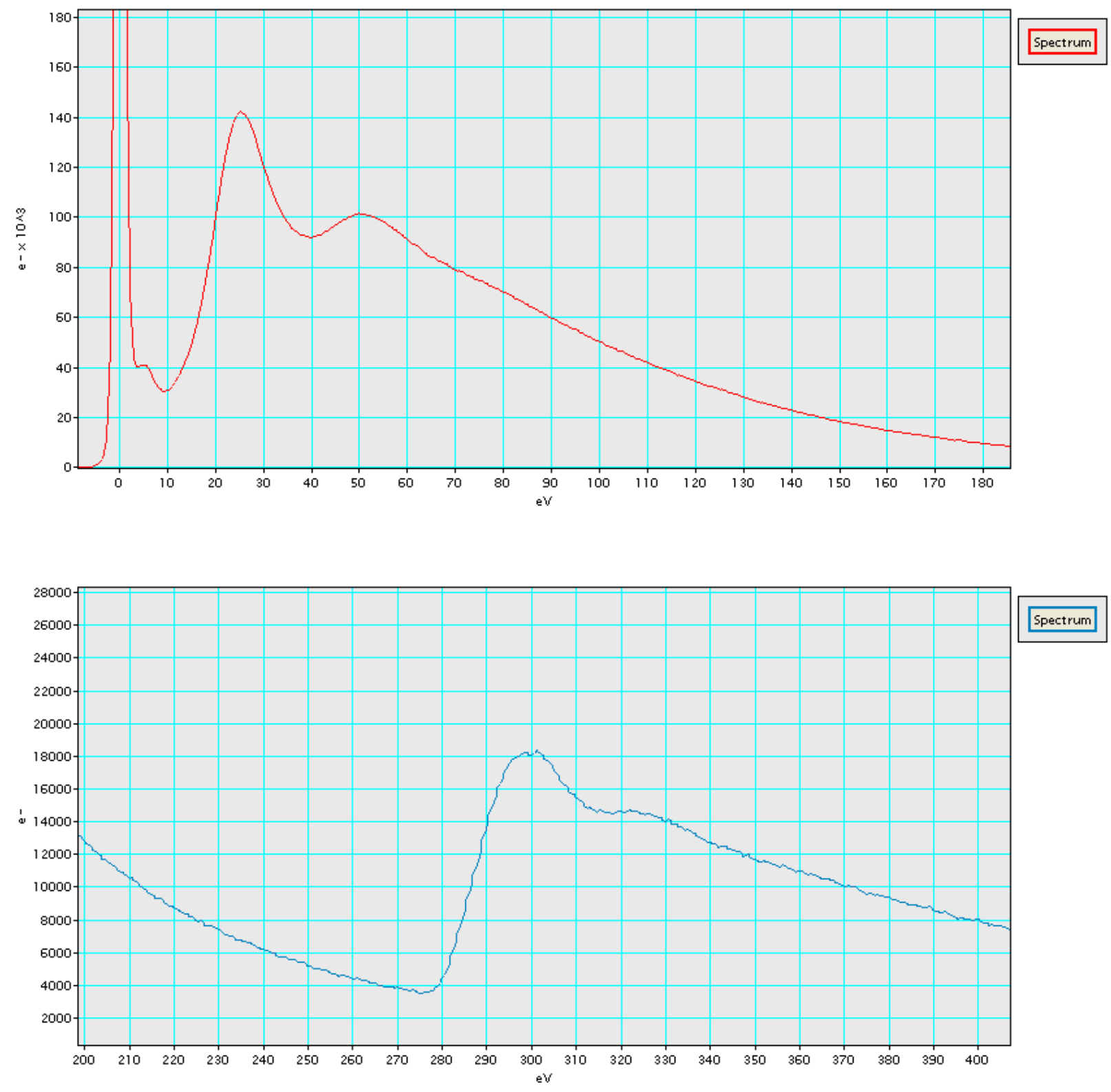

Figure 3S: EELS spectra corresponding to Li and $\mathrm{C}$ mappings in Li-RGO sample (Primary energy: $200 \mathrm{keV}$; Dispersion: $0.3 \mathrm{eV} /$ pixel; Energy shift: $0 \mathrm{eV}$; Adjust: $-2.9 \mathrm{eV}$ ).

\section{References:}

[1]. Celzard, J. F.; Mareche, G.; Furdin, Prog. Mater. Sci. 2004, 50, 93

[2].Chung, D. D. L. J. Mater. Sci. 2002, 37, 1475

[3]. Lee, S.; Cho, D.; Drzal, L. T. J. Mater. Sci. 2005, 40, 2001. 